\title{
Entropy spectrum of a Kerr anti-de Sitter black hole
}

\author{
Deyou Chena 11 and Haitang Yang 2 \\ ${ }^{a}$ Institute of Theoretical Physics, China West Normal University, \\ Nanchong, 637009, China \\ ${ }^{b}$ Department of Physics, Sichuan University, Chengdu, 610065, China
}

\begin{abstract}
The entropy spectrum of a spherically symmetric black hole was derived without the quasinormal modes in the work of Majhi and Vagenas. Extending this work to rotating black holes, we quantize the entropy and the horizon area of a Kerr anti-de Sitter black hole by two methods. The spectra of entropy and area are obtained via the Bohr-Sommerfeld quantization rule and the adiabatic invariance in the first way. By addressing the wave function of emitted (absorbed) particles, the entropy and the area are quantized in the second one. Both results show that the entropy and the area spectra are equally spaced.
\end{abstract}

\section{Introduction}

Quantum effects of black holes are important topics. It is widely believed that the horizon area of black holes ought to be quantized. In 1970s, Bekenstein first quantized the horizon area and derived the expression of area spectrum as $A_{n}=n \gamma l_{p}^{2}$, where $\gamma$ is a dimensionless constant, $l_{p}$ is the Planck length and $n=1,2,3 \cdots$. In his work, the black hole is non-extreme and the horizon is regarded as a classical adiabatic invariant [1]. Inspired by this work, people quantized the horizon area by different methods and derived the area spectrum which has the same form as that obtained by Bekenstein.

One of the methods relies on quasinormal modes (QNMs), where QNMs is defined by the solution of the perturbational wave equation when a classical black hole is perturbed by an exterior field. The quasinormal mode frequency plays an important role in the quantum properties of black holes, especially in the quantization of the horizon area. Considering this frequency denotes characteristics of black holes, Hod employed the Bohr's correspondence principle to quantize the horizon area and found that the area spectrum is related to the real part of QNMs [2]. For general non-extreme black holes, the area spacing is $\Delta A=4 l_{p}^{2} \ln 3$. This value is in consistence with both statistical physics arguments and the area-entropy thermodynamic relation. Latter

\footnotetext{
${ }^{1}$ E-mail: ruijianchen@gmail.com

2 E-mail: hyanga@uestc.edu.cn
} 
on, Dreyer and Kunstatter investigated the area spectra from loop quantum gravity and the semi-classical arguments, respectively [3, 4]. In Dreyer's work [3], from the relation between QNMs and the entropy spectrum, the Immirzi parameter was fixed to $\gamma=\frac{\ln 3}{2 \sqrt{2} \pi}$. In Kunstatter's work [4], the vibrational frequency $(\omega(E))$ of quasinormal mode is related to the energy $(E)$ of a black hole. Then the quantity $I=\int \frac{d E}{\omega(E)}$ is an adiabatic invariant and has the equally spaced spectrum via the Bohr-Sommerfeld quantization rule. Applying this quantity to higher-dimensional black holes, he derived equally spaced area spectrum and entropy spectrum of a D-dimensional Schwarzschild black hole. The area spectra of Reissner-Nordstrom black holes, BTZ black holes and Schwarzschild-de Sitter black holes can be referred to [5].

Hod's work is interesting, but there are some points needed to be improved. First of all, the research on the asymptotic behavior of the quasinormal mode frequencies of Kerr and Reissner-Nordstrom black holes shows the constant $4 \ln 3$ is not a universal value. Secondly, in Hod's derivation, he only discussed transitions from the ground state to a state with large $n$. When two arbitrary states are taken into account, the area changes would be an arbitrarily small value. The above problems were fixed in [6]. It turns out that a classical black hole perturbed by an exterior field has the same behavior as that of a damped harmonic oscillator. Maggiore proved the physical frequency of QNMs is determined by both its real part and imaginary part. He further put forward that the area spectrum oughts to be determined by the asymptotic value of the physical frequency [6]. Applying this new explanation to the quantization of the horizon area, he derived the equally spaced area spectrum as $A_{n}=8 n l_{p}^{2}$. This value is different from that derived by Hod, but in consistence with the result obtained by Bekenstein. Using this new explanation, many progresses discussing the area spectra of black holes arose [7, 18, 9, 10, 11, 12, 13, 14, 15, 16, 17, 18, 19, 20, 21, 22].

One method quantizing the entropy via the adiabatic invariance and the Bohr-Sommerfeld quantization rule was put forward by Majhi and Vagenas [23. In their work, the adiabatic invariant quantity connects to the Hamiltonian of black holes. Using the Hamiltonian and the Bohr-Sommerfeld quantization rule, they derived the equally spaced spectrum of entropy of a spherically symmetric black hole. However, whether the horizon area spectrum is equally spaced or not relies on the relation between the horizon area and the entropy. The area spectrum is equally spaced when the area is proportional to the entropy. Or, it is not equally spaced. In [33], with the observation of 
the periodicity of the outgoing waves, another way was proposed to quantize the entropy. The periodicity is exhibited by the periodic property in the wave function. One interesting point is that QNMs is not required in both methods.

In this paper, we adopt two ansatz to quantize the entropy and the horizon area of a Kerr anti-de Sitter black hole. Our work in the first ansatz is an extension of the work of Majhi and Vagenas. The Bohr-Sommerfeld quantization rule and the adiabatic invariance are introduced. In the second ansatz, the wave function of the emitted (absorbed) particles plays an important role. Then the entropy and the area are quantized by the wave function. Both results show the entropy spectrum and the horizon spectrum are respectively equally spaced.

The rest is organized as follows. In Sect. 2, we derive the Hamiltonian of the Kerr anti-de Sitter black hole with the emission of a particle. The quantization of the entropy and the horizon area via the Bohr-Sommerfeld quantization rule is dicussed. In Sect. 3, the relation between the HamiltonJacobi equation and the Klein-Gordon equation is reviewed and the entropy and the area are quantized by the wave function of particles in the black hole's background spacetime. Sect. 4 contains discussion and conclusion.

\section{Quantize the entropy via the adiabatic invariance}

In this section, the entropy and the horizon area of the Kerr anti-de Sitter black hole are quantized via the the adiabatic invariance and the BohrSommerfeld quantization rule. The Kerr anti-de Sitter metric is given by [24]

$$
\begin{aligned}
d s^{2}= & -\frac{\Delta}{\rho^{2}}\left(d t-\frac{a}{\Xi} \sin ^{2} \theta d \varphi\right)^{2}+\frac{\Delta_{\theta} \sin ^{2} \theta}{\rho^{2}}\left(a d t-\frac{r^{2}+a^{2}}{\Xi} d \varphi\right)^{2} \\
& +\frac{\rho^{2}}{\Delta} d r^{2}+\frac{\rho^{2}}{\Delta_{\theta}} d \theta^{2}
\end{aligned}
$$

where

$$
\begin{aligned}
\Delta & =\left(r^{2}+a^{2}\right)\left(1+r^{2} l^{-2}\right)-2 M r, \quad \Xi=1-a^{2} l^{-2} \\
\rho^{2} & =r^{2}+a^{2} \cos ^{2} \theta, \quad \Delta_{\theta}=1-r^{2} l^{-2} \cos ^{2} \theta
\end{aligned}
$$

$a$ stands for the angular momentum per unit mass and $l$ is a constant related to the cosmological constant $\Lambda=-3 l^{-2}$. The event horizon is located at 
$r=r_{+}$, determined from $\Delta=0$. The Hawking temperature and the angular velocity at the event horizon are respectively

$$
\Omega_{+}=\frac{a \Xi}{r_{+}^{2}+a^{2}}, \quad T=\frac{r_{+}+r_{+}\left(3 r_{+}^{2}+a^{2}\right) l^{-2}-a^{2} r_{+}^{-1}}{4 \pi\left(r_{+}^{2}+a^{2}\right)} .
$$

The Bohr-Sommerfeld quantization rule says

$$
\int p_{i} d q_{i}=n h
$$

where $p_{i}$ is the conjugate momentum of the coordinate $q_{i}$ with $i=0,1, \cdots$. This is an adiabatic invariant quantity and $n=1,2,3 \cdots$. To quantize the horizon area, one can first Euclideanize the metric. The Euclideanized metric was derived by the coordinate transformation $t \rightarrow-i \tau$ and $a \rightarrow i a$ for a general rotating spacetime [25]. In this paper, we first perform the dragging coordinate transformation, and then Euclideanize the Kerr anti-de Sitter metric. To achieve this purpose, let $d \varphi=-\frac{g_{03}}{g_{33}} d t$, the metric becomes

$$
d s^{2}=-\frac{\Delta \Delta_{\theta} \rho^{2}}{\Delta_{\theta}\left(r^{2}+a^{2}\right)^{2}-\Delta a^{2} \sin ^{2}} d t^{2}+\frac{\rho^{2}}{\Delta} d r^{2}+\frac{\rho^{2}}{\Delta_{\theta}} d \theta^{2} .
$$

Then the Euclideanized Kerr anti-de Sitter metric is obtained by a transformation $t \rightarrow-i \tau$. To quantize the horizon area, we connect the adiabatic invariant quantity to the parameters of the black hole. The adiabatic invariant quantity is rewritten as

$$
\int p_{i} d q_{i}=\iint_{0}^{H} \frac{d H^{\prime}}{\dot{q}_{i}} d q_{i}=\iint_{0}^{H} d H^{\prime} d \tau+\iint_{0}^{H} \frac{d H^{\prime}}{\dot{x}_{\mu}} d x_{\mu} .
$$

Now $q_{i}$ 's denote the coordinates of the Euclideanized Kerr anti-de Sitter spacetime. $q_{0}=\tau$ is the Euclidean time, which has a periodicity $T_{0}=$ $\frac{2 \pi}{\kappa}$ and $\kappa$ is the surface gravity of the black hole [26]. $x_{\mu}$ 's stand for the spacelike coordinates with $\mu=1,2$, and $x_{1}=q_{1}=r, x_{2}=q_{2}=\varphi \cdot H$ represents the Hamiltonian of the system and satisfies $H=\int_{\tau_{i}}^{\tau_{f}} L d \tau$, with $L$ being the Lagrangian function. Due to the rotation of the black hole, there exists a frame dragging effect of the coordinate system in the rotating space-time. The matter field in the ergosphere near the horizon must be 
dragged by the gravitational field with an azimuthal angular velocity. So the Hamiltonian should reflect this effect. From the function, we find that $\varphi$ is a cyclic coordinate and its degree of freedom ought to be eliminated. Therefore the Hamilton function is written as

$$
H=\int_{\tau_{i}}^{\tau_{f}}\left(L-P_{\varphi} \dot{\varphi}\right) d \tau=\int_{r_{i}}^{r_{f}} \int_{0}^{P_{r}} d P_{r}^{\prime} d r-\int_{\varphi_{i}}^{\varphi_{f}} \int_{0}^{P_{\varphi}} d P_{\varphi}^{\prime} d \varphi,
$$

where $P_{r}$ and $P_{\varphi}$ are canonical momenta of $r$ and $\varphi \cdot r_{i}$ and $r_{f}$ are locations of the event horizons before and after the particle is emitted, respectively. Different particles have different equations of motion. For a massless particle, its equation of motion is the null radial geodesics $\dot{r}=\frac{d r}{d \tau}[27]$. While the equation of motion is the phase velocity of the particle for a massive one, namely $\dot{r}=v_{p}$ [28]. In this section, we only consider the outgoing path and don't care the concrete expression of the path in detail. Thus it does not affect our result whether the particle is massless or not [29]. The thermodynamic property of the Kerr anti-de Sitter black hole has been deeply investigated. The expressions of Hamilton canonical equations can be derived as

$$
\begin{aligned}
& \dot{r}=\left.\frac{d H}{d P_{r}}\right|_{\left(r ; \varphi, P_{\varphi}\right)},\left.\quad d H\right|_{\left(r ; \varphi, P_{\varphi}\right)}=d M^{\prime} ; \\
& \dot{\varphi}=\left.\frac{d H}{d P_{\varphi}}\right|_{\left(\varphi ; r, P_{r}\right)},\left.\quad d H\right|_{\left(\varphi ; r, P_{r}\right)}=\Omega^{\prime} d J^{\prime} ;
\end{aligned}
$$

where $\Omega^{\prime}$ and $\dot{r}$ are the angular velocity and the equation of motion of the emitted particle. Using Eq. (7), we can rewrite Eq. (마) as

$$
\begin{aligned}
H & =\int_{r_{i}}^{r_{f}} \int_{0}^{H} \frac{\left.d H^{\prime}\right|_{\left(r ; \varphi, P_{\varphi}\right)}}{\dot{r}} d r-\int_{\varphi_{i}}^{\varphi_{f}} \int_{0}^{H} \frac{\left.d H^{\prime}\right|_{\left(\varphi ; r, P_{r} ;\right)}}{\dot{\varphi}} d \varphi \\
& =\left.\int_{0}^{\tau_{0}} \int_{0}^{H} d H^{\prime}\right|_{\left(r ; \varphi, P_{\varphi}\right)} d \tau-\left.\int_{0}^{\tau_{0}} \int_{0}^{H} d H^{\prime}\right|_{\left(\varphi ; r, P_{r}\right)} d \tau \\
& =\int_{0}^{\tau_{0}} \int_{0}^{H} d H^{\prime} d \tau .
\end{aligned}
$$


Now the adiabatic invariant quantity connected to the parameters of the black hole is taken on

$$
\begin{aligned}
\int p_{i} d q_{i} & =2 \int_{0}^{\tau_{0}} \int_{0}^{H} d H^{\prime} d \tau \\
& =2\left[\int_{0}^{\tau_{0}} \int_{0}^{M} d M^{\prime} d \tau-\int_{0}^{\tau_{0}} \int_{0}^{J} \Omega^{\prime} d J^{\prime} d \tau\right] .
\end{aligned}
$$

Here we only consider the outgoing path. This implies we only select the half value of periods of the Euclidean time and then $\tau_{0}=\frac{\pi}{\kappa}$. Meanwhile from the first law of thermodynamics of the Kerr anti-de Sitter black hole

$$
d M=T d S+\Omega_{0} d J
$$

where $\Omega_{0}$ expresses the angular velocity at the event horizon, we finish the integral and get

$$
\int p_{i} d q_{i}=2 \int_{0}^{S} \tau_{0} \cdot T d S^{\prime}=\hbar S
$$

The last equality in the above equation was obtained by the relation between the surface gravity and the Hawking temperature $T=\frac{\hbar \kappa}{2 \pi}$. Using the BohrSommerfeld quantization rule, we get the black hole entropy as

$$
S=2 \pi n
$$

It shows that the entropy is discrete and the minimal spacing of the entropy spectrum is $\Delta S=S_{n}-S_{n-1}=2 \pi$, which is irrelevant to the parameters of the Kerr anti-de Sitter black hole. From the area-entropy relation $S=\frac{A}{4 l_{p}^{2}}$, the horizon area spectrum is obtained as

$$
A=8 \pi n l_{p}^{2}
$$

which implies the minimal spacing of the area spectrum is $\Delta A=8 \pi l_{p}^{2}$. Therefore, the entropy spectrum and the area spectrum are respectively equally 
spaced and irrelevant to the parameters of the black hole.

\section{Quantize the entropy via the wave function}

In this section, the entropy and the horizon area are quantized via the wave function of the particle in the Kerr anti-de Sitter black hole's background spacetime. The wave function can be obtained from the Dirac equation or the Klein-Gordon equation. Here we choose the Klein-Gordon equation. Its connection to the Hamilton-Jacobi equation was investigated in Ref. [30]. We first review this connection. The Klein-Gordon equation for a scalar field is given by

$$
g^{\mu \nu} \partial_{\mu} \partial_{\nu} \Phi-\frac{m^{2}}{\hbar^{2}} \Phi=0
$$

Use the WKB approximation and let the wave function have the following form

$$
\Phi\left(t, x^{i}\right)=\exp \left[\frac{i}{\hbar} I\left(t, x^{i}\right)\right]
$$

Inserting this expression into Eq. (14) and multiplying both sides by $\hbar^{2}$ yield

$$
g^{\mu \nu} \partial_{\mu} I \partial_{\nu} I+m^{2}=0
$$

which is just the Hamilton-Jacobi equation and was used to investigate the tunnelling radiation of scalar particles and fermions [30, 31]. The fermion tunnelling from the Kerr anti-de Sitter black hole was investigated and the action $I$ of a massive (or massless) particle was derived in 32]

$$
I=-(\omega-j \Omega) t+W(r)+j \phi+\Theta(\theta),
$$

where $\omega=-\partial_{t} I$ is the energy of the emitted particle measured by an observer at infinity and $j=\partial_{\varphi} I$ is the angular quantum number about $\varphi$. Inserting this action into Eq. (15), we get

$$
\Phi\left(t, x^{i}\right)=\exp \left\{\frac{i}{\hbar}[-(\omega-j \Omega) t+W(r)+j \phi+\Theta(\theta)]\right\}
$$




$$
\begin{gathered}
=\exp \left[-\frac{i}{\hbar}(\omega-j \Omega) t\right] \cdot \exp \left[\frac{i}{\hbar} W(r)\right] \\
\cdot \exp \left(\frac{i}{\hbar} j \phi\right) \cdot \exp \left[\frac{i}{\hbar} \Theta(\theta)\right] .
\end{gathered}
$$

Near the horizon, $W(r)= \pm i \pi \frac{r_{+}^{2}+2 b r_{+}+a^{2}}{2\left(r_{+}-M\right)}\left(\omega-j \Omega_{+}\right)$is related to the Hawking temperature, where the $+(-)$ express the outgoing (ingoing) paths. In Ref. 33, the authors described the wave function in the Schwarzschild and Kerr spacetimes and pointed out the function $\phi$ and $\Theta(\theta)$ would vanish near the corresponding horizons. However, from the wave function, we find they will not vanish and have certain values, though these values will not affect our result. Furthermore, it can be found that there are periodicities of $t, \phi$ and $\Theta(\theta)$ from the expression of $\Phi\left(t, x^{i}\right)$. The periods of them are respectively

$$
T_{0}=\frac{h}{\left(\omega-j \Omega_{+}\right)}, \quad \phi_{0}=\frac{h}{j}, \quad \Theta_{0}(\theta)=h .
$$

The loss of a black hole system's energy is related to the physical frequency, as addressed in Ref. [6]. In this section, we connect the system's energy to the frequency of the wave function and let $E=\omega-j \Omega_{+}=\hbar \omega_{0}$, where $\omega_{0}$ is the frequency of the wave function. From Eq. (19), we get the relation between the periods and the frequency as $T_{0}=\frac{2 \pi}{\omega_{0}}$. Furthermore, it should be noted that the wave function $\Phi\left(t, x^{i}\right)$ contains both the outgoing and the ingoing solutions. When we consider the black hole emits (absorbs) a particle with energy $\omega$ and angular momentum $j$, the changes of energy and angular momentum of this black hole are related to these of the particle, namely $d M=\omega, d J=j$. From the first law of thermodynamics of this black hole, we get

$$
d S=\frac{1}{T}\left(d M-\Omega_{+} d J\right)=\frac{1}{T}\left(\omega-j \Omega_{+}\right)=\frac{1}{T} \frac{2 \pi \hbar}{T_{0}} .
$$

$T$ is the temperature of the black hole, which is related to the surface gravity as $T=\frac{\hbar \kappa}{2 \pi}$. Meanwhile, the periodic surface gravity is given by $\kappa=\frac{2 \pi}{T_{0}}$ [26]. Thus we get the change of the black hole's entropy as

$$
\Delta S=2 \pi
$$

which shows the minimal value of change of the Kerr anti-de Sitter black hole's entropy is $2 \pi$. This result is full in consistence with that gotten in 
section 2. Using the same process adopted in section 2 , we can also get the minimal spacing of the horizon area spectrum as $\Delta A=8 \pi l_{p}^{2}$.

\section{Discussion and Conclusion}

In this paper, we have quantized the entropy and the horizon area of the Kerr anti-de Sitter black hole by two methods. In the first one, the spectra of entropy and area were derived by the adiabatic invariance and the BohrSommerfeld quantization rule. The adiabatic invariant quantity connects to the Hamiltonian of the black hole. In the second ansatz, the wave function of the particle plays an important role. The entropy was quantized by the wave function. Both results are fully in consistence with each other and show that the entropy spectrum and the area spectrum are respectively equally spaced. These results are in consistence with that obtained by Bekenstein and that derived by Maggiore. In this paper, we used the area-entropy relation

$S=\frac{A}{4 l_{p}^{2}}$, so the area spectrum was derived and equally spaced. However, the area is not proportional to black hole entropy in other theories, such as Loop quantum gravity [34]. When we consider this situation, the area spectrum may not be equally spaced.

\section{Acknowledgements}

This work is supported in part by the Natural Science Foundation of China (Grant No.11178018 and No. 11175039).

\section{References}

[1] J. D. Bekenstein, The quantum mass spectrum of the Kerr black hole. Lett. Nuovo Cimento 11467 (1974).

[2] S. Hod, Bohr's correspondence principle and the area spectrum of quantum black holes. Phys. Rev. Lett., 814293 (1998).

[3] O. Dreyer, Quasinormal modes, the area spectrum, and black hole entropy. Phys. Rev. Lett., 90081301 (2003).

[4] G. Kunstatter, D-dimensional black hole entropy spectrum from quasi-normal modes. Phys. Rev. Lett., 90161301 (2003). 
[5] M. R. Setare, Area spectrum of extremal Reissner-Nordstrom black holes from Quasi-normal modes. Phys. Rev. D 69044016 (2004).

M. R. Setare, Non-rotating BTZ black hole area spectrum from Quasi-normal modes. Class. Quant. Grav., 211453 (2004).

M. R. Setare, Near extremal Schwarzschild-de Sitter black hole area spectrum from Quasi-normal modes. Gen. Rel. Grav., 371411 (2005).

[6] M. Maggiore, The physical interpretation of the spectrum of black hole quasinormal modes. Phys. Rev. Lett., 100141301 (2008).

[7] E. C. Vagenas, Area spectrum of rotating black holes via the new interpretation of quasinormal modes. J. High Energy Phys., 0811073 (2008).

[8] A. J. M. Medved, On the Kerr quantum area spectrum. Class. Quant. Grav., 25205014 (2008).

[9] D. Kothawala, T. Padmanabhan, S. Sarkar, Is gravitational entropy quantized? Phys. Rev. D 78104018 (2008).

[10] R. Banerjee, B. R. Majhi, E. C. Vagenas, Quantum tunneling and black hole spectroscopy. Phys. Lett. B 686279 (2010).

[11] A. Lopez-Ortega, Area spectrum of the D-dimensional de Sitter spacetime. Phys. Lett. B 68285 (2009).

[12] S. Fernando, Spinning dilaton black holes in 2+1 dimensions: Quasi-normal modes and the area spectrum. Phys. Rev. D 79124026 (2009).

[13] K. Ropotenko, Quantization of the black hole area as quantization of the angular momentum component. Phys. Rev. D 80044022 (2009).

[14] S. W. Wei, R. Li, Y. X. Liu, J. R. Ren, Quantization of black hole entropy from quasinormal modes. J. High Energy Phys., 0903076 (2009).

[15] Y. Kwon, S. Nam, Area spectra of the rotating BTZ black hole from quasinormal modes. arXiv:1001.5106 [hep-th]

[16] Y. S. Myung, Area spectrum of slowly rotating black holes. arXiv: 1003.3519 [hep-th]

[17] D. Chen, H. Yang, X. T. Zu, Area spectra of near extremal black holes. Eur. Phys. J. C 69289 (2010). 
[18] M. R. Setare, E. C. Vagenas, Area spectrum of Kerr and extremal Kerr black holes from Quasinormal modes. Mod. Phys. Lett. A 201923 (2005).

[19] E. Berti, V. Cardoso, A. O. Starinets, Quasinormal modes of black holes and black branes. arXiv:0905.2975 [gr-qc]

[20] S. W. Wei, Y. X. Liu, K. Yang, Y. Zhong, Entropy/Area spectra of the charged black hole from quasinormal modes. Phys. Rev. D 81104042 (2010).

[21] W. Li, L. Xu, J. Lu, Area spectrum of near-extremal SdS black holes via the new interpretation of quasinormal modes. Phys. Lett. B 676177 (2009).

[22] P. Gonzalez, E. Papantonopoulos, J. Saavedra, Quasi-normal modes of scalar perturbations, mass and area spectrum of Chern-Simons black holes. J. High Energy Phys., 08050 (2010).

[23] B. R. Majhi, E. C. Vagenas, Black hole spectroscopy via adiabatic invariance. Phys. Lett. B 701623 (2011).

[24] B. Carter, Killing horizons and orthogonally transitive groups in space-time. Commun. Math. Phys., 10280 (1968).

[25] Z. Z. Ma, Euler numbers of four-dimensional rotating black holes with the Euclidean signature, Phys. Rev. D 67 024027, (2003).

[26] G. W. Gibbons, M. J. Perry, Black holes and thermal Green functions. Proc. R. Soc. Lond A 358467 (1978).

[27] M. K. Parikh, F. Wilczek, Hawking radiation as tunneling. Phys. Rev. Lett., 855042 (2000).

[28] J. Y. Zhang, Z. Zhao, Hawking radiation of charged particles via tunneling from the Reissner-Nordstrom black hole. JHEP 10055 (2005).

[29] K. Umetsu, Hawking radiation from Kerr-Newman black hole and tunneling mechanism. Int. J. Mod. Phys. A 254123 (2010).

[30] R. Kerner, R. B. Mann, Fermions tunnelling from black holes. Class. Quant. Grav., 25095014 (2008).

[31] R. Kerner, R. B. Mann, Charged fermions tunnelling from Kerr-Newman black holes. Phys. Lett. B 665277 (2008). 
[32] D. Chen, H. Yang, X. T. Zu, Fermion tunneling from anti-de Sitter spaces. Eur. Phys. J. C 56119 (2008).

[33] X. X. Zeng, X. M. Liu, W. B. Liu, Periodicity and area spectrum of black holes. arXiv:1203.5947[gr-qc].

[34] R. K. Kaul, P. Majumdar, Logarithmic correction to the Bekenstein- Hawking entropy. Phys. Rev. Lett., 845255 (2000). 